\title{
Energy Dissipation and Shear Rate with Geometry of Baffled Surface Aerator
}

\author{
Bimlesh Kumar* \\ Department of Civil Engineering, Indian Institute of Technology Guwahati, Guwahati-781039, India
}

Received 02 May 2010; received in revised form 28 July 2010

\begin{abstract}
The geometric parameters of surface aeration systems govern the process phenomena. The dissipation rate of turbulent kinetic energy $(\varepsilon)$ and shear rate $(\gamma)$ are the key process parameters for mixing in surface aerators. Present work by doing numerical computation by visimix ${ }^{\circledR}$ analyzes the effect of geometric parameters (impeller diameter, cross-sectional area of the tank, liquid height, width of the baffle, rotor blade length and immersion height) on $\varepsilon$ and $\gamma$. Analysis has been done by making the geometric parameters non-dimensionalized through rotor diameter. With an increase in liquid height and baffled width, there is an increase in the case of energy dissipation and shear rate values. In the case of tank area and blade length, it is vice versa. Energy dissipation and shear rate are not affected by the variation in immersion height of the impeller.
\end{abstract}

Keywords: energy dissipation, mixing, shear rate, surface aerator

DOI:10.3329/cerb.v14i2.4910

\section{Introduction}

Aeration is one of the important processes employed in water and wastewater treatment to reduce biochemical oxygen demand. The basic phenomenon behind the process of aeration is a gas transfer, in which gas molecules are exchanged between the liquid and the gas at the gas-liquid interface [1]. Many types of aerators are used in practice, such as cascade, spray nozzles, diffused or bubble aerators and surface aerators. Among them surface aerators are popular because of their comparable efficiency and ease in operation [2-4]. Surface aeration systems are most frequently used in aerobic biological reactors to supply microorganisms with oxygen and to mix the fermentation broth [5]. Surface aerators are very effective in supporting the growth of cells in suspension and on micro-carriers [6]. Surface aeration is a commonly used mechanism to supply oxygen to mammalian cells [7-9].

The geometry governs the aeration process. Efficient aeration performance can be achieved by understanding the effect of geometry on it. By rotating the impeller, kinetic energy has been imparted to the fluid, which dissipated through viscous dissipation. This viscous dissipation is supposed to happen at very low scale of turbulence. This low scale of turbulence is generally governed by the Kolmogorov's laws of isotropy. Thus, it is needed to quantify the dissipation rate of the turbulence kinetic energy $(\varepsilon)$ in surface aerators [10-13]. The second aspect is shear rate, which is mainly controlled by the shear produced by the impeller. The determination of shear rate in surface aerators is an important step in deciding their suitability to handle shear sensitive bio-systems. High shear fields resulting from the fluid physical properties and the hydrodynamics may cause damage to fragile microorganisms and bio-films formation $[14,15]$ or mechanical instability to immobilized biocatalysts $[16,17]$. However, a

$\overline{\text { *Corresponding author, Email }}$ :bimk@iitg.ernet.in certain degree of shearing is required to attain sufficient heat and mass-transfer rates, and to achieve a homogeneous distribution of transferred components into the bulk fluid.

Thus, the goal of this paper is to investigate the effect of various geometric factors on energy dissipation and shear rate in surface aeration systems.

\section{Theory}

Figure 1 shows the schematic drawing of a baffled surface aeration tank used in the present study. The geometric variables include cross-sectional area of the tank $(A)$, depth of water in the tank $(H)$, diameter of the rotor $(D)$, width of the baffle $(B)$, length of the blades $(l)$, width of the blades $(b)$, distance between the top of the blades and the horizontal floor of the tank $(h)$ and the number of blades $(n)$ as shown in Figure 1. In the present study, $n$ is constant and equals to 6 and the ratio of $l / b$ has been maintained constant $(=1.25)$. The geometric parameters have been non-dimensionalized by dividing through the rotor diameter (D). Energy dissipation rate $(\varepsilon)$ and shear rate $(\gamma)$ on different geometric parameters have been calculated by using visimix ${ }^{\circledR}$ [18] software at different impeller rotational speed.

\subsection{Dissipation Rate of Turbulent Kinetic Energy, $\varepsilon$}

The turbulent kinetic energy can be used to characterize the flow at a scale small compared to that of the mean motion. The energy dissipation rate, , is an important characteristic of the flow and mixing at a scale where the molecular transport processes become dominant [19]. This process is generally governed by the Kolmogorov's laws [19]. In turbulent flow, instability of the main flow amplifies existing disturbances and produces primary eddies which have a wave length or scale similar to that of the main flow. The large primary eddies are also unstable and disintegrate into smaller and smaller eddies until all their energy is dissipated 


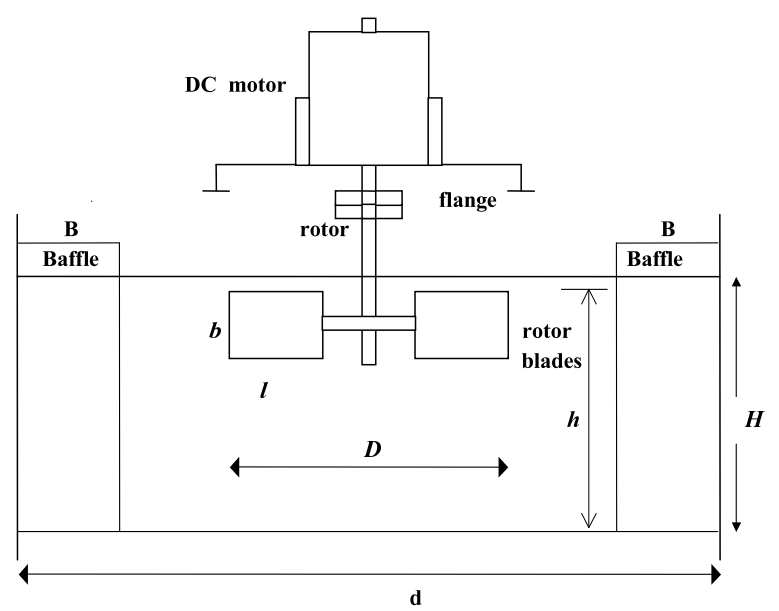

Figure 1: Schematic diagram of a Surface Aeration Tank

by viscous flow. These eddies transfer kinetic energy from the large to small eddies, and because this transfer occurs in different directions, directional information of the large eddies is gradually lost. The question now arises as to how the energy dissipation rate can be measured. The direct measurement of is very difficult, since it needs to capture precisely the smallest turbulent structures [20]. One of the main uncertainties is over the method employed and the approximations necessarily made to estimate the local dissipation rate of turbulent kinetic energy. Extensive studies have been carried out on the subject and the approaches can be broadly divided into three main methods:

- Kinetic energy balance term averaged over a control volume [21-23]

- Integration of dissipation spectrum [24, 25]

- Dimensional analysis [26]

In the present work, of surface aeration systems has been calculated by using commercial software Visimix ${ }^{\circledR}$ [18]. The Visimix ${ }^{\circledR}$ [18] program can be helpful in analyzing the mixing parameters in a stirred tanks $[27,28]$. The calculation procesure (which is kinetic energy balance term averaged over a control volume) are described as follows:

The mean value of the kinetic energy of turbulence, $E$ at the radius $r$ is defined as:

$$
E=3 \bar{v}^{2} / 2
$$

where $\bar{v}^{\prime}$ is the mean square root velocity of turbulent pulsations corresponding to the largest local linear scale of turbulence. Steady-state transport of the turbulent component of kinetic energy can be described as:

$$
q\left(\frac{d E}{d r}\right)-\frac{d}{d r}\left[2 \pi r h_{j} v_{e}\left(\frac{d E}{d r}\right)\right]+2 \pi r h_{j} \varepsilon=0
$$

where $q$ is the circulation flow rate through the rotor, $v_{e}$ is the eddy viscosity and $h_{j}$ is the local linear scale of turbulence which is approximately equal to $1.5 b$. Equation 2 is solved for $\bar{v}^{\prime}=0$ at $r=\infty$. The value of $\bar{v}^{\prime}$ on the other boundary $(r=d / 2)$ is calculated using an estimated value of the maximum dissipation rate in the flow past the blades:

$$
\varepsilon_{m}=\left[\left(2 \pi r N-v_{o}\right) \operatorname{Sin} \alpha\right]^{3} / l
$$

where $v_{o}$ is the axial velocity of the rotor. The dimensions of the $\varepsilon_{m}$ zone (length, height and width) are $l, b$ and $b / 2$, respectively. The mean value of dissipation is estimated as:

$$
\varepsilon=\varepsilon_{m} N b / 6 \pi r
$$

The unit of turbulent dissipation rate, $\varepsilon$ is $W / \mathrm{kg}$. Equation 4 is solved numerically to get the value of $\varepsilon$ at different rotational speed.

\subsection{Shear Rate}

Shear rate influences power consumption, mixing characteristics and mass transfer phenomena in surface aerators. The specific energy dissipation rate in a stirred tank is well known to depend on the shear rate $\gamma$ and the shear stress $\tau$ [29], as follows [30]:

$$
\gamma=\sqrt{\frac{1}{\mu} \frac{P}{V}}
$$

Where $P$ is the power input, $\mu$ is dynamic viscosity of the fluid and $V$ is the volume of the fluid in the tank. Equation 5 applies to laminar, turbulent and transitional flows. In laminar ranges, $\gamma$ is linearly related to the rotational speed of the rotor $(N)$ [31]. In turbulent flow, it is nonlinearly related to $N$ [30]. One of the simpler ways to calculate shear rate is agitator tip speed over the distance between the tip and the vessel wall:

$$
\gamma=N D /(d-D)
$$

where $d$ is the tank diameter. Visimix ${ }^{\circledR}$ [18] however, defines shear rate as the ratio of turbulent fluctuation velocity, $v_{0}$, to the Kolmogorov turbulence scale, $L_{0}$ as follows:

$$
\gamma=v_{0} / L_{0}
$$

$L_{0}$ is called the Kolmogorov length and depends primarily upon the power input per unit mass and the kinematic viscosity. Values of shear rate calculated at different geometric parameters at different rotational speeds.

\section{Results and Discussion}

The purpose of the present work is to find the effect of geometric parameters on $\varepsilon$ and $\gamma$. The values of $\varepsilon$ and $\gamma$ are calculated at different values of non-dimensional geometric variables at different speed as discussed above.

\subsection{Effect Due to Variation in $H / D$}

In the present work, the value of non-dimensionalized $H / D$ has been varied from 0.8 to 1 to find out the energy dissipating trend in the vicinity of the impeller. Kresta and Wood [32] investigated turbulence quantities in a stirred vessel and assumed that the bulk of the energy is contained in the largest eddies, the flow is at local equilibrium and the flux of 

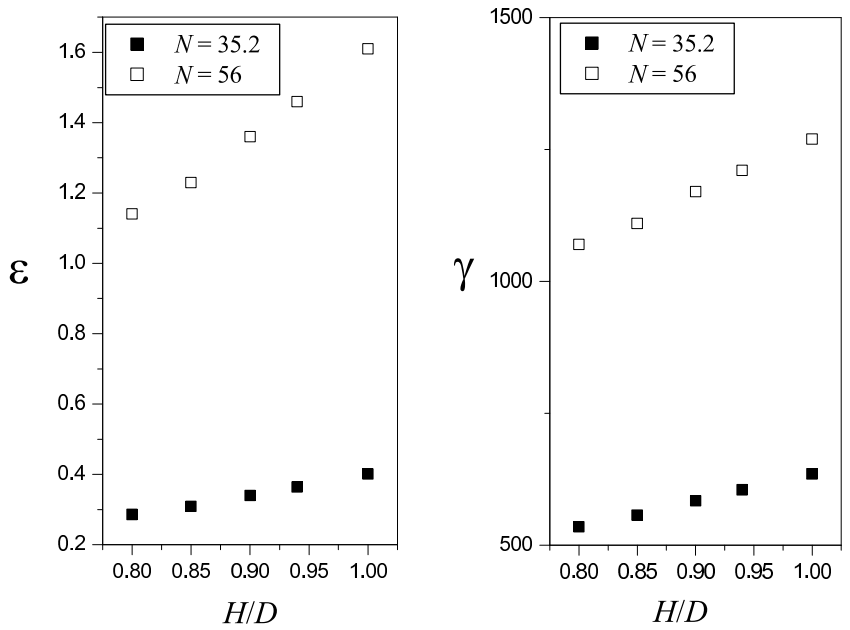

Figure 2: Effect on $\varepsilon$ and $\gamma$ due to variations in $H / D$

energy towards smaller scales is constant. Zhou and Kresta [26] used a dimensional argument and found that most of the total energy is dissipated in the near vicinity of the impeller discharge. The results have been shown in the Figure 2. As shown in the Figure 2, both and show an increasing trend. It clearly indicates that the increase of rotational speed will increase the liquid pumping capacity of the impeller, thus the mixing quality will be improved and consequently energy dissipation and shear rate.

\subsection{Effect Due to Variable Width of Baffles}

Installation of the baffles effectively destroys the circular liquid patterns, inhibiting the vortex formation so that the liquid surface becomes almost flat [33, 34]. Moreover, axial flows become much stronger, leading to an improved mixing rate. For these reasons, baffled tanks are the more widely used in industrial applications. Standard baffle number in circular tank is said to 4 , which is called as a condition of complete baffling of the tank [29]. By putting standard number of baffles, non-dimensional width of the baffle has been varied from $0.2 D$ to $0.8 D$. Results have been shown in the Figure 3. As shown in the Figure 3 , increasing $B / D$ shows an increment in $\varepsilon$ and $\gamma$. The reason for this behavior may be attributed for more power is required at higher values of $B / D$.

\subsection{Effect Due to Variation in $h / D$}

It has been found that and are unaffected the variation in $h / D$, which signifies the impeller submergence as shown in the Figure 4. Here, it can be hypothesized that such behavior is due to the fact that by definition of the surface aeration systems, impeller should be placed as near as water surface. Preserving the definition of surface aerator, there is no significant variations in $h / D$. The given variations are not able to effect the energy dissipation and shear rate significantly.

\subsection{Effect Due to Variation in $l / D$}

The geometry of the rotor blade affects of hydrodynamics of the surface aeration systems. It affects not only the power
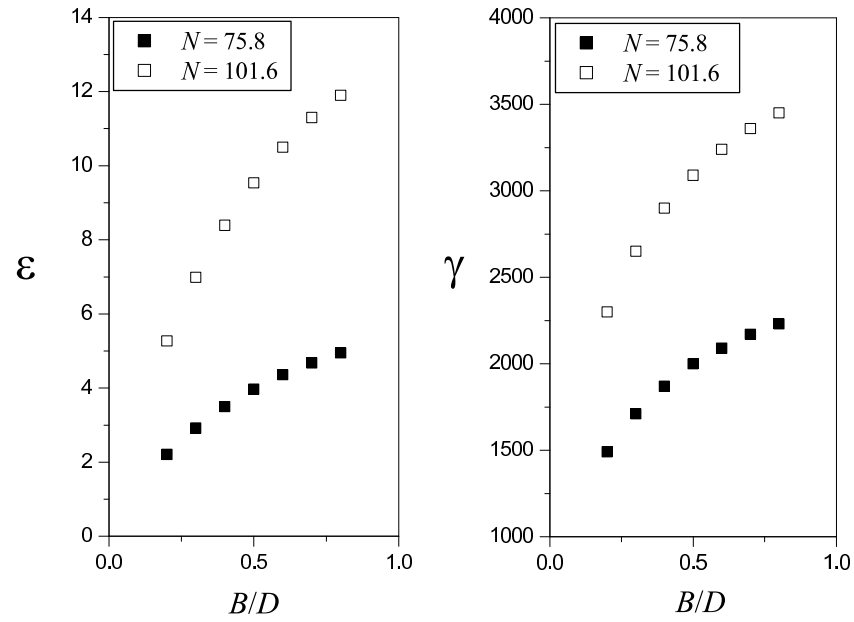

Figure 3: Effect on $\varepsilon$ and $\gamma$ due to variable $B / D$
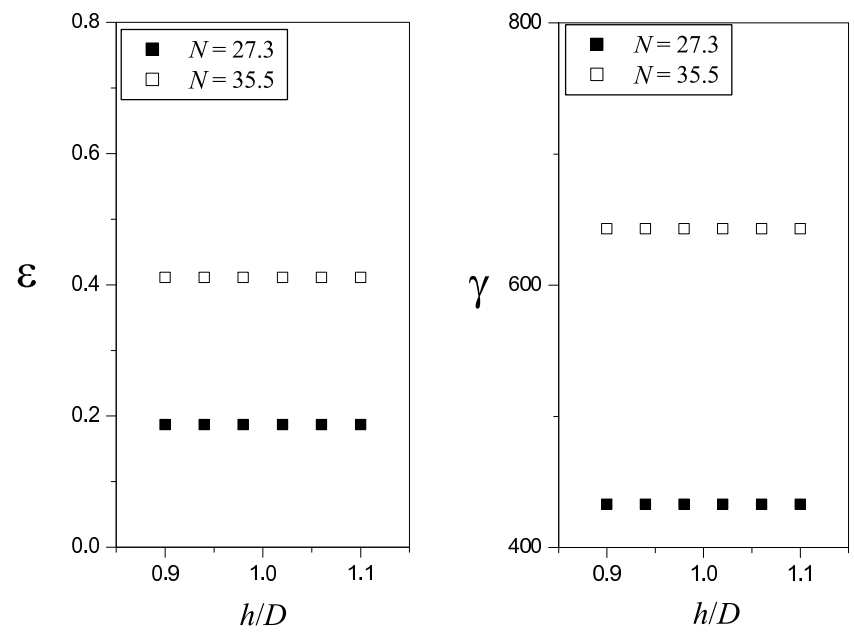

Figure 4: Effect on $\varepsilon$ and $\gamma$ due to variations in $h / D$

consumption, but also the flow patterns. To ascertain the effect of blade geometry on $\varepsilon$ and $\gamma, l / D$ has been varied from 0.25 to 0.36 . Results have been shown in the Figure 5 .

It can be seen from the Figure 5 that an increases in blade width have been seen to result in corresponding decrease in $\varepsilon$ and $\gamma$.

\subsection{Effect Due to Variation in $\sqrt{A / D}$}

Trends of $\varepsilon$ and $\gamma$ due to variation in $\sqrt{A / D}$ have been shown in the Figure 6. It shows a decreasing with increasing $\sqrt{A / D}$. The reason for such can be attributed to the fact that a lower value of $\sqrt{A / D}$ intensifies the turbulence, which results in high energy dissipation and shear rate.

\section{Conclusion}

The rate of dissipation of the turbulence energy $(\varepsilon)$ and shear rate $(\gamma)$ signifies the process characteristics of surface aeration systems. Thus, it is important to quantify the variation of the rate of dissipation of the turbulence energy $(\varepsilon)$ and 

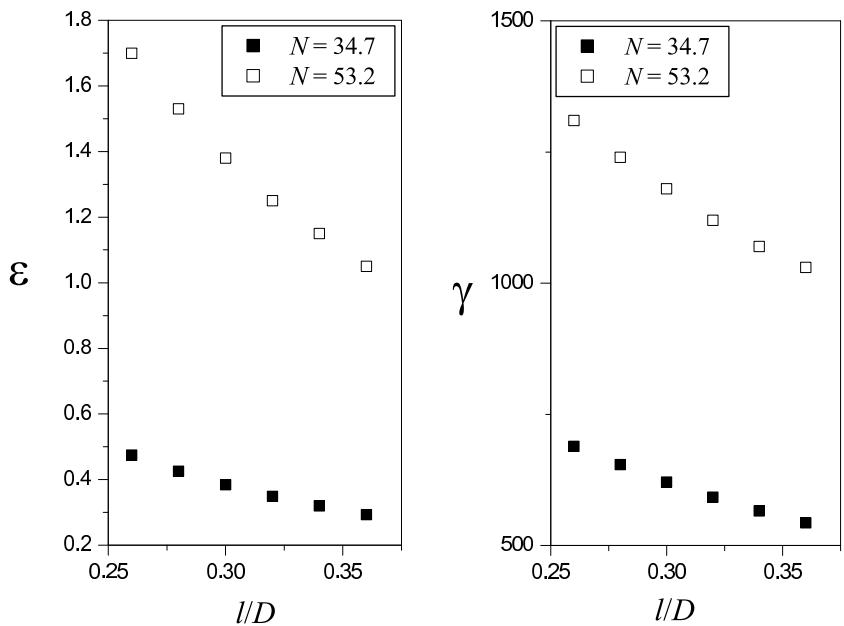

Figure 5: Effect on $\varepsilon$ and $\gamma$ due to variations in $l / D$
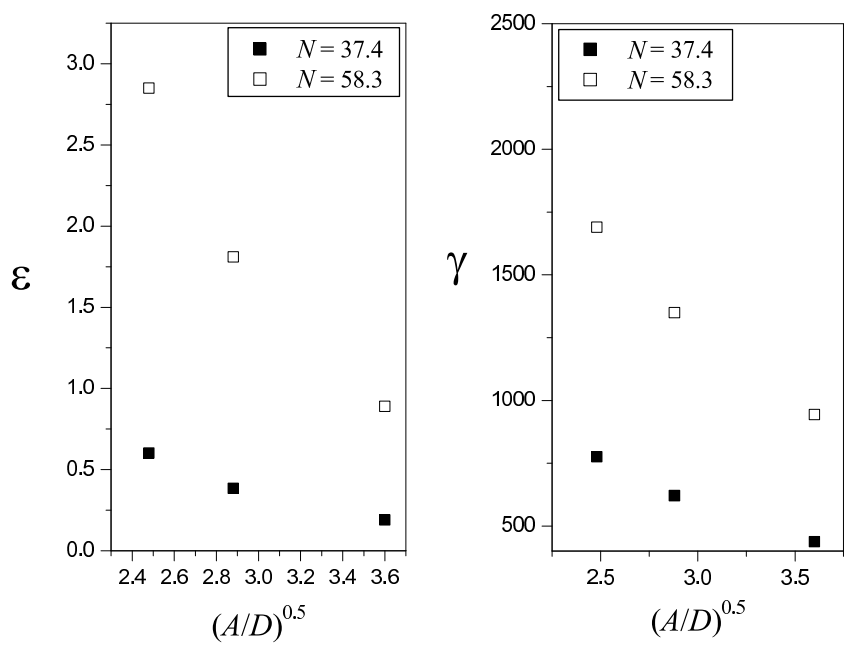

Figure 6: Effect on $\varepsilon$ and $\gamma$ due to variations in $\sqrt{A / D}$

shear rate $(\gamma)$ with geometric parameters in surface aeration systems, which may be useful in the design and operation of surface aeration systems. Present work shows qualitatively how the variations in geometric parameters affect the $\varepsilon$ and $\gamma$. It is found that with an increase in $H / D$ and $B / D, \varepsilon$ and $\gamma$ increases whereas it is vice versa in case of $l / D$ and $\sqrt{A / D}$.

\section{References}

[1] Fair G, Gayer J and Okum D, Elements of water supply and waste water disposal, John Wiley and Sons, 1967

[2] McWhirter JR, Chern JM and Hutter JC, Oxygen Mass Transfer Fundamentals of Surface Aerators, Industrial \& Engineering Chemistry Research, 1995. 34(8):pp. 2644-2654. doi:10.1021/ie00047a013

[3] Rao AR and Kumar B, Scale-up criteria of square tank surface aerator, Biotechnology and Bioengineering, 2007. 96(3):pp. 464-470. doi: $10.1002 /$ bit. 21149

[4] Rao A and Kumar B, Scaling Up of the Geometrically Similar Unbaffled Circular Tank Surface Aerators, Chemical Engineering \& Technology, 2008. 31(2):pp. 287-293. doi : 10.1002 / ceat. 200700300

[5] Hu WS, Meier J and Wang DIC, Use of surface aerator improve oxygen transfer in cell culture, Biotechnology and Bioengineering, 1986. 28(1):pp. 122-125. doi:10.1002/bit.260280120
[6] Ognean T, Dimensionless criteria for estimating oxygen transfer in aeration systems, Biotechnology and Bioengineering, 1993. 41(11):pp. 1014-1020. doi:10.1002/bit.260411103

[7] Bliem R, Konopitzky K and Katinger H, Bioreactor Systems and Effects, Ch. Industrial animal cell reactor systems: Aspects of selection and evaluation, Advances in Biochemical Engineering/Biotechnology, Springer, 1991. pp. 1-26, doi:10.1007/Bfb0000746. doi:10.1007/Bfb0000746

[8] Kawase Y and Moo-Young M, Volumetric mass transfer coefficients in aerated stirred tank reactors with Newtonian and non-Newtonian media, Chemical Engineering Research and Design, 1988. 66:pp. 284288

[9] Gilbertson JA, Sen A, Behie LA and Kallos MS, Scaled-up production of mammalian neural precursor cell aggregates in computercontrolled suspension bioreactors, Biotechnology and Bioengineering, 2006. 94(4):pp. 783-792. doi:10.1002/bit.20900

[10] Yianneskis M, Popiolek Z and Whitelaw JH, An experimental study of the steady and unsteady flow characteristics of stirred reactors, Journal of Fluid Mechanics, 1987. 175:pp. 537-555. doi:10.1017/S002211208700051X

[11] Schäfer M, Höfken M and Durst F, Detailed LDV Measurements for Visualization of the Flow Field Within a StirredTank Reactor Equipped with a Rushton Turbine, Chemical Engineering Research and Design, 1997. 75(8):pp. 729-736. doi:10.1205/026387697524399

[12] Yianneskis M and Whitelaw J, On the structure of the trailing vortices around Rushton turbine blades, Chemical Engineering Research and Design, 1993. 71(a):pp. 543-550

[13] Kumar B and Rao AR, Oxygen transfer and energy dissipation rate in surface aerator, Bioresource Technology, 2009. 100(11):pp. 28862888. doi:10.1016/j.biortech.2008.12.031

[14] Gjaltema A, Tijhuis L, van Loosdrecht MCM and Heijnen JJ, Detachment of biomass from suspended nongrowing spherical biofilms in airlift reactors, Biotechnology and Bioengineering, 1995. 46(3):pp. 258269. doi:10.1002/bit.260460309

[15] Gjaltema A, van der Marel N, van Loosdrecht MCM and Heijnen JJ, Adhesion and biofilm development on suspended carriers in airlift reactors: Hydrodynamic conditions versus surface characteristics, Biotechnology and Bioengineering, 1997. 55(6):pp. 880-889

[16] Leenen EJTM, Dos Santos VAP, Grolle KCF, Tramper J and Wijffels $\mathrm{R}$, Characteristics of and selection criteria for support materials for cell immobilization in wastewater treatment, Water Research, 1996. 30(12):pp. 2985-2996. doi:10.1016/S0043-1354 (96) 00209-6

[17] dos Santos VAPM, Leenen EJTM, Rippoll MM, van der Sluis C, van Vliet T, Tramper J and Wijffels RH, Relevance of rheological properties of gel beads for their mechanical stability in bioreactors, Biotechnology and Bioengineering, 1997. 56(5):pp. 517-529

[18] A Review of the Main Mathematical Models Used in the VisiMix ${ }^{\circledR}$ Software, VisiMix Ltd.: http://www.visimix.com

[19] Pope S, Turbulent Flows, Cambridge University Press, Cambridge, 2000

[20] Saarenrinne P and Piirto M, Turbulent kinetic energy dissipation rate estimation from PIV velocity vector fields, Experiments in Fluids, 2000. 29(0):pp. S300-S307. doi:10.1007/s003480070032

[21] Cutter LA, Flow and turbulence in a stirred tank, AIChE Journal, 1966. 12(1):pp. 35-45. doi:10.1002/aic.690120110

[22] Wu H and Patterson G, Laser-Doppler measurements of turbulent-flow parameters in a stirred mixer, Chemical Engineering Science, 1989. 44(10):pp. 2207-2221. doi:10.1016/0009-2509 (89) 85155-3

[23] Zhou G and Kresta S, Distribution of energy between convective and turbulent flow for three frequently used impellers, Chemical Engineering Research and Design, 1996. 74(a):pp. 379-389

[24] Okamoto Y, Nishikawa M and Hashimoto K, Energy dissipation rate distribution in mixing vessels and its effects on liquid-liquid dispersion and solid-liquid mass transfer, International Chemical Engineering, 1981. 21(1):p. 88Ü94

[25] Costes J and Couderc J, Study by laser Doppler anemometry of the turbulent flow induced by a Rushton turbine in a stirred tank: Influence of the size of the units-I. Mean flow and turbulence, Chemical Engineering Science, 1988. 43(10):pp. 2751-2764. doi:10.1016/0009-2509(88) 80018-6

[26] Zhou G and Kresta SM, Impact of tank geometry on the maximum turbulence energy dissipation rate for impellers, AIChE Journal, 1996. 
42(9):pp. 2476-2490. doi:10.1002/aic.690420908

[27] Liu K and Neeld K, Simulation of mixing and heat transfer in stirred tanks with VisiMixö Solutia Inc., in Topical Conference on Process Development from Research to Manufacturing: Industrial Mixing and Scale-up, AICHE Annual Meeting, Dallas, TX, 1999

[28] Genck W, Optimizing crystallizer scale up, Chemical Engineering Progress, 2003. 99:pp. 36-44

[29] Nagata S, Mixing Principles and applications, John Wiley \& Sons, 1975

[30] Sánchez Pérez J, Rodríguez Porcel E, Casas López J, Fernández Sevilla J and Chisti Y, Shear rate in stirred tank and bubble column bioreactors, Chemical Engineering Journal, 2006. 124(1-3):pp. 1-5. doi:10.1016/j.cej.2006.07.002

[31] Metzner AB and Otto RE, Agitation of non-Newtonian fluids, AIChE Journal, 1957. 3(1):pp. 3-10

[32] Kresta SM and Wood PE, The flow field produced by a pitched blade turbine: Characterization of the turbulence and estimation of the dissipation rate, Chemical Engineering Science, 1993. 48(10):pp. 17611774. doi:10.1016/0009-2509(93) 80346-R

[33] Ciofalo M, Brucato A, Grisafi F and Torraca N, Turbulent flow in closed and free-surface unbaffled tanks stirred by radial impellers, Chemical Engineering Science, 1996. 51(14):pp. 3557-3573. doi:10.1016/0009-2509(96)00004-8

[34] Lu WM, Wu HZ and Ju MY, Effects of baffle design on the liquid mixing in an aerated stirred tank with standard Rushton turbine impellers, Chemical Engineering Science, 1997. 52(21-22):pp. 38433851. doi:10.1016/S0009-2509(97) 88929-4 